\title{
ON CURRENT PENETRATION AND PLASMA DISRUPTION
}

BY

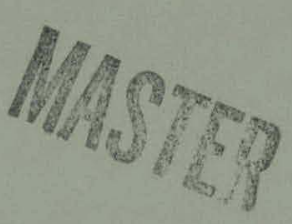

T. H. STIX

\section{PLASMA PHYSICS LABORATORY}

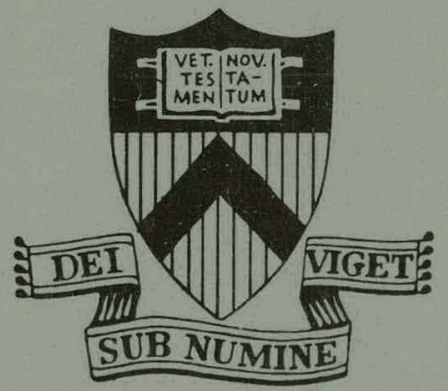

\section{PRINCETON UNIVERSITY PRINCETON, NEW JERSEY}

This work was supported by U. S. Energy Research and Development Administration Contract E(11-1)-3073. Reproduction, translation, publication, use and disposal, in whole or in part, by or for the United States Government is permitted. 


\section{DISCLAIMER}

This report was prepared as an account of work sponsored by an agency of the United States Government. Neither the United States Government nor any agency Thereof, nor any of their employees, makes any warranty, express or implied, or assumes any legal liability or responsibility for the accuracy, completeness, or usefulness of any information, apparatus, product, or process disclosed, or represents that its use would not infringe privately owned rights. Reference herein to any specific commercial product, process, or service by trade name, trademark, manufacturer, or otherwise does not necessarily constitute or imply its endorsement, recommendation, or favoring by the United States Government or any agency thereof. The views and opinions of authors expressed herein do not necessarily state or reflect those of the United States Government or any agency thereof. 


\section{DISCLAIMER}

Portions of this document may be illegible in electronic image products. Images are produced from the best available original document. 
NOTICE

This report was prepared as an account of work sponsored by the United States Government. Neither the United States nor the United States Energy Research and Development Administration, nor any of their employees, nor any of their contractors, subcontractors, or their employees, makes any warranty, express or implied, or assumes any legal liability or responsibility for the accuracy, completeness or usefulness of any information, apparatus, product or process disclosed, or represents that its use would not infringe privately owned rights.

Printed in the United States of America.

Available from

National Technical Information Service

U. S. Department of Commerce 5285 port Royal Road

Springfield, Virginia 22151

Price: Printed Copy $\$$ * ; Microfiche $\$ 1.45$

*Pages

$$
\begin{gathered}
1-50 \\
51-150 \\
151-325 \\
326-500 \\
501-1000
\end{gathered}
$$

NTIS

Selling Price

$\$ 4.00$

5.45

7.60

10.60

13.60 
On Current Penetration and Plasma Disruption

\author{
Thomas H. Stix \\ Plasma Physics Laboratory \\ Princeton University \\ Princeton, New Jersey, 08540
}

This reporice

This report was prepared as an account of work the United the United Siates Government. Neither Research and Deves not the United States Energy their employees, subcontractors. or their en their contractors. warranty, express or implied, or assumakes any Lability of responsibility for the accuracy, uny legal proserulness of any information, apparatus, product process disclosed, or represents that its use would of fringe privately owned resents that its use would not

It is suggested that, in a tokamak discharge, the initial rapid current penetration and the disruptive instability may each be attributed to a type of nonlinear double tearing instability which causes growth and overlap of radially-separated magnetic islands. The ensuing reconnection and braiding of magnetic lines would enhance local radial heat and particle transport, and could permit rapia redistribution of plasma current, inducing positive or negative spikes in the loop-voltage signal.

Two curious characteristics of tokamak discharges are the initial-phase current penetration, ${ }^{1}$ which appears to proceed anomalously fast, and the later-phase disruptive instability,? which shows negative spikes on the loop voltage signal and is characteristically followed by rapid plasma movement and loss. We suggest that the two processes are related, namely, that both are instances of growth and overlap of sets of magnetic islands on adjacent rational surfaces. Such overlap would bring about relatively rapid reconnection of magnetic lines of force and could cause sudden redistribution of the plasma current: The associated rapid change of magnetic flux would produce positive 
or negative voltage spikes and the magnetic-line reconnection would enhance radial heat, and particle transport. ${ }^{3}$

Two models for island overlap are proposed. The more tractable one involves the helically-symmetric double tearing mode ${ }^{4}$ in its nonlinear evolution. In this mode, perturbations grow at each of two singular $(\vec{k} \cdot \vec{B}=0)$ surfaces in the plasma. The rotational transform must be the same at the two surfaces, but this condition may in fact arise during the skin phase of the tokamak discharge when the plasma current at the core is stil]. low. If, in the nonlinear-growth phase of this instability, the islands overlap, reconnection of the magnetic lines and a new magnetic topology may be expected to appear. Because this mode retains helical symmetry, the magnetic surfaces are not destroyed but they may become highly convoluted. Redistribution of plasma current along the new surfaces would then appear as fast radial current penetration.

In addition to its application to understanding anomalous current penetration, the nonlinear double tearing mode is of interest for computer modelling of the tokamak plasma. ${ }^{5}$ The mode can be studied in cylindrical geometry with helical symmetry and exact magnetic surfaces, and its evolution with reconnection of magnetic surfaces and rapid current redistribution might also reproduce the major characteristics of the disruptive instability.

The second model for island overlap pertains to the normal phase of the tokamak discharge, when the rotational transform is a monotonically decreasing function of the plasma minor radius. We consider the presence of a primary set of islands at a rational 
surface, slowly growing due to instability of the ordinary tearing mode. In the magnetostatic analysis, nonlinear mixing of the primary perturbation mode [mode numbers $\mathrm{m}, \mathrm{n} ; \mathrm{q}(\mathrm{r})=\mathrm{m} / \mathrm{n}$ ] with the toroidal geometric factor, $\mathrm{R}+\mathrm{rcos} \theta,(i . e ., \mathrm{m}= \pm 1, \mathrm{n}=0$ ) produces associated perturbation magnetic fields ${ }^{6}$ with helicity $m \pm 1, n$. Satellite islands must therefore appear at $q(r)=$ $(m \pm 1) / n$ with widths expected to be of the order of $(r / R)^{1 / 2}$ times the width of the primary islands. A qualitative discussion indicates that the same mechanism which leads to nonlinear growth for the double tearing mode can enhance the growth of the outer satellites and speed up the approach to overlap. We suggest that the disruptive instability is due to the occurrence of such accelerated growth and overlap.

From an analytical point of view, the interaction between islands of incommensurate helicity is very different from the case of the double tearing mode with helical symmetry. The approach to island overlap in the asymmetric case produces local stochastic destruction of the magnetic surfaces and braiding of the magnetic lines of force in this region. 7,8 Throughout the braided region the current density (more accurately, $j_{n / B}$ ) and electron temperature must become uniform.

To understand the generation of voltage spikes, let us consider plasma currents, differing in magnitude, flowing on adjacent magnetic surfaces. If the magnetic field lines are somehow suddenly braided, transient phenomena will occur, after which $j_{1 /} / B$ in this region, as just mentioned, is uniform. simplifying the model to the interaction of just two sheet currents, an 
electric-circuit analogy, Fig. 1 , is helpful, particularly in view of the difficulties cited in the literature $^{2}$ in accounting for the polarity of the spikes. Initially, current $i_{1}$ flows on the inner of two adjacent toroidal magnetic surfaces, and current $i_{2}, i_{2} \neq i_{1}$, on the outer. The energy of the poloidal magnetic field stored between the two surfacè is represented by $L_{i}{ }^{i}{ }_{1}^{2} / 2$, $L_{i}$ symbolizing the "leakage inductance." similarly, the magnetic energy stored outside the outer surface is represented by $\left(\mathrm{Li}_{1}{ }^{2}+2 \mathrm{Mi}_{1} \mathrm{i}_{2}+\mathrm{Li}_{2}\right)^{2} / 2$. Braiding intermixes the field lines, effectively creating the series electrical connection illustrated in the right-hand diagram in Fig. 1. At first, the current through the inductive elements is still $i_{1}$ and $i_{2}, i_{1} \neq i_{2}$, and $\nabla \cdot j \simeq 0$ requires that the difference current, $i_{2}-i_{1}$, flow radialiy between the former surfaces. The likely physical process for this transient radial flow is polarization current, producing poloidal plasma motion which would be quickly dissipated, primarily in ion heat. 9,10 The radial current path is represented electrically by some (high) impedance $z$, bridging the inductive elements. Circuit analysis with perfect coupling, $L=M$, and with a resistive impedance for $\mathrm{z}$ shows a net change, $\Delta i$, in the total loop current, $\Delta i=2 i_{\text {final }}-\left(i_{1}+i_{2}\right) \simeq\left(L_{i} / 4 L\right)\left(i_{1}-i_{2}\right)$ and also shows that an energy approximately $L_{i}\left(i_{1}-i_{2}\right)^{2} / 8$ is dissipated in the bridging resistance. Thus, if the outer sheet current ( $\left.i_{2}\right)$ were initially greater than the inner sheet current ( $\left.{ }_{1}\right)$.' as in the skin phase for a tokamak discharge, current penetration by braiding would result in a slightly smaller total loop current and would be accompanied by a sudden reduction of the external 
poloidal magnetic field, giving rise to a positive voltage spike on the loop-voltage signal. 11 on the other hand, for $i_{1}>i_{2}$ initially, as in the configuration preceding a normal disruption, the voltage spike would be negative.

We turn now to the consideration of the double tearing mode, under which tearing of magnetic lines occurs in the vicinity of each of two nearby magnetic surfaces which have the same value of $\vec{k} \cdot \vec{B}$ or rotational transform. Now it is, in fact, simpler to offer an example of a multiple tearing mode. We consider the force-free equilibrium,

$$
\vec{B}_{0}(x)=\hat{z}\left(B^{2}+J^{2} \lambda^{-2} \sin ^{2} \lambda x\right)^{1 / 2}+\hat{y} J \lambda^{-1} \cos \lambda x
$$

and follow the classic analysis ${ }^{12}$ of the tearing instability with perturbations of the form $f_{I}(\vec{r}, t)=f_{I}(x) \exp (i k y+\gamma t)$. Away from the singular $\left(\vec{k} \cdot \vec{B}_{0}=0\right)$ surfaces, the perturbation $\psi \equiv \mathrm{B}_{\mathrm{x}}{ }^{(1)} / \mathrm{B}$ closely follows the infinite-conductivity relation, Eq. (20) of Ref. (12), namely, $\nabla^{2} \psi=\psi F^{-1} d^{2} F / d x^{2}$, with $F \equiv \mathrm{B}_{\mathrm{Y}}{ }^{(0)} / \mathrm{B}$. With $\vec{B}_{\mathrm{O}}(\mathrm{x})$ as given, the simple solution $\psi \sim \cos :\left[\left(\lambda^{2}-k^{2}\right)^{1 / 2} x\right]$ exp $(i k y+\gamma t)$ satisfies the infiniteconductivity perturbation equation, and Fig. 2 shows how the $\psi$ solutions may be chosen in successive outer regions. The jump in $d \psi / \psi \bar{d} x$ at each singular surface is $(\Delta / / a) \equiv d \psi_{+} / \psi_{+} d x-$ $d \psi_{-} / \psi_{-} d x=2\left(\lambda^{2}-k^{2}\right)^{1 / 2} \tan \left[\left(\lambda^{2}-k^{2}\right)^{1 / 2} \pi / 2 \lambda\right]$ which is matched to the inner-region resistive solutions (dotted lines in Fig. 2) and is related to the instability growth rate via Eqs. $(34),(36)$ and (49) of Ref. (12),

$$
\gamma=\left(\frac{k^{2} J^{2}}{81 \pi \rho}\right)^{1 / 5}\left(\frac{\Delta^{\prime}}{a}\right)^{4 / 5}\left(\frac{n}{4 \pi}\right)^{3 / 5}
$$


The mode is unstable for $|k|<|\lambda|$ [and probably unstable for all $|k|=\left|k_{y}^{2}+k_{z}^{2}\right|^{1 / 2}$, choosing $k_{z}$ such that singular surfaces occur at $\lambda x=2 \ell \pi \pm \varepsilon, \quad l$ integral, with $\varepsilon$ sufficiently small, cf. Sec. V, Ref. (4)], showing growth at all singular surfaces and showing the same $\operatorname{sign}$ for $\psi \equiv \mathrm{B}_{\mathrm{X}}{ }^{(1)} / \mathrm{B}$ for $y=$ constant, all $x$. The new magnetic surfaces, including now the first-order perturbations, display thin islands around the former singular surfaces $(\lambda x=\ell \pi+\pi / 2)$. Because the sign of the magnetic shear changes. with. $\lambda(\Delta x)=\pi$, adjacent island chains show a $180^{\circ}$ shift of spatial phase.

Growth of the multiple tearing mode may be expected to continue through its nonlinear phase. Developing a physical picture of this nonlinear growth, we first note that for the force-free plasma, $\vec{j}(\vec{r})=\gamma(\vec{r}) \vec{B}(\vec{r})$, and $\nabla \cdot \vec{j}=0$ implies $\vec{B} \cdot \nabla \gamma=0, i . e .$, $j_{z} / B_{z}$ is constant along each of the $\gamma=$ constant magnetic surfaces. Now in our multiple-mode equilibrium; $(4 \pi / \mathrm{c}) j_{z}(0)=$ $\mathrm{dB}_{\mathrm{y}}{ }^{(0)} / \mathrm{dx}=-\mathrm{J} \sin \lambda \mathrm{x}$ and $j_{\mathrm{z}}(0)$ would be alternately positive and negative on magnetic surfaces of adjacent island chains. Diffusion tends to smooth out such differences by normal resistive (eddy current) processes. Adopting for the moment a viscous model of resistive dissipation, our intuition would picture current dif-fusing across the magnetic surfaces at rates proportional to the local gradients, but simultaneously spreading out along each surface to maintain constancy of $j_{z} / B_{z}$. Now as current diffuses away from the positive-j $(0)$ islands, the current deficit appears mainly in the region of the $x$-stagnation points just because the magnetic surfaces are spread apart most widely there. 
It is easy to verify that the perturbation magnetic field due to this deficit is properly phased in space to enlarge the islands. Similarly, the current accumulations at the $x$-points of the negative-j ${ }_{2}{ }^{(0)}$ chains are seen also to contribute to island enlargement as one recalls that the magnetic shear is reversed in adjacent layers. Hopefully, computer analysis will provide quantitative support for this intuitive description of island growth.

Looking now at the nonlinear growth of the double tearing mode, the sketches in Fig. 3 represent bald guesses at the possible evolution of the magnetic surfaces for this instability, and are modelled with the simple function $\psi=\left(x^{3} / 3\right)-x_{0}{ }^{2}+$ $\varepsilon(t) \cos k y$, the perturbation-size parameter, $\varepsilon$, increasing from the top to the bottom sketch. Of particular interest is the reconnection of the flux surfaces that occurs at intermediate $\varepsilon$. If such reconnection were to take place in the double tearing mode, it would be accompanied by a rapid redistribution of the current and electron heat along the new convoluted and extended magnetic surfaces.

For normal-phase tokamak operation, the rotational transform is a monotonically decreasing function of radius, and the equilibrium configuration is not appropriate to the two-dimensional double tearing instability: On the other hand, toroidal effects will cause satellite islands to be associated with any set of primary islands. The reason is qualitatively clear -- the primary islands are produced by a helical radial perturbation magnetic 
field: This perturbation field is, in turn, induced by a perturbation current, and it is this current which must have the same helicity as the local magnetic field $(e \cdot g \cdot, \vec{B} \cdot \nabla \gamma=0)$. Solving then for the induced perturbation $\vec{B}$ field, the magnetostatic equations introduce nonlinear mixing of the primary perturbation field, $B_{r}{ }^{(1)} \sim b(r) \cos (m \theta-n \phi)$, with the toroidal geometry factor, $l+(r / R) \cos \theta$, so that secondary fields of order, $\Delta B_{d}{ }^{(1)} \sim(r / R) b(r) \cos [(m \pm 1) \theta-n \phi]$, etc., appear as necessary components of the complete self-consistent solution. Associated with primary. $q=2$ islands will therefore be $q=3$ satellite islands with width $\sim(r / R)^{1 / 2}$ times as large as the primary island. Second-harmonic current components in the primary island (e.g., $m=4, n=2$ ) can similarly lead to satellites at $q=3 / 2$ and $q=5 / 2$. $q=3 / 2$ satellites would also be induced by second-harmonic components $(m=2, n=2)$ in a primary $q=1$ plasma current distortion.

One suggestion might then be that plasma disruption occurs when primary islands due, say, to a single tearing mode, grow sufficiently to overlap with their own satellite islands or, perhaps, with the $q=3 / 2$ satellites of a $q=1$ core distortion. But under certain conditions we could also expect growth of the satellite islands themselves. Specifically, in a geometry with both radially decreasing $j_{\|}$and transform, diffusion toward and accumulation of $j_{n}$ near the $x$-point regions of outer satellite islands would produce perturbation. $\vec{B}$ fields with proper spatial phase to enhance the width $\sim\left[\mathrm{B}_{r}{ }^{(1)}\right]^{1 / 2}$ of these islands (at 
some minor cost to the width of the larger primary-island). We could expect the combined system to be unstable with characteristic growth rates for the outer satellites typical of the nonlinear tearing mode, 13 and this instability of the combined system, with satelite growth and island overlap speeded up by $x$-point current accumulation, suggests a quite interesting mechanism for sudden plasma disruption.

\section{ACKNOWLEDGEMENTS}

It is a pleasure to acknowledge helpful discussions with Dr. A. Rechester and with Drs. K. Bol, P. H. Kutherford, J. D. Strachan, and S.. von Goeler.

*This work was supported by U. S. Energy Research and Development Administration Contract $\mathrm{E}(11-1)-30 \% 3$. 
REFERENCES

I see, for example, D. F. Duchs, H. P. Furth, P. H. Rutherford, Nucl. Fusion 12, 341 (1972), and references cited therein.

2 See, for example, H. P. Furth, Nucl. Fusion 15, 487 (1975), and references cited therein.

T. H. Stix, Phys. Rev. Letters 30, 833 (1973).

4 H. P. Furth, P. H. Rutherford, and H. Selbery, Pliys. l'luida 16, 1054 (1973).

5 Computer analysis of the nonlinear single tearing mode has recently been carried out by B. V. Waddell, D. A. Monticello, M. N. Rosenbluth, and R. B. White, Proc. Seventh Conf. on Numerical Simuiation, Cousanl Inst., N. Y. U., Juno 1975, p. 2.

6 T. H. Stix, Bull. Am. Phys. Soc. 18,1367 (1973).

7 M. N. Rosenbluth, R. Z. Sagdeev, J. B. Taylor, and G. M. Zaslavski, Nucl. Fusion 6 , 297 (1966).

8 A. Rechester and T. H. Stix, MATT-1168, Plasma Physics Laboratory, Princeton, iv. J. 08540 , August 1975 .

9 M. N. Rosenbluth, P. H. Ruthertord, J. B. Taylur, E. A. Frieman, and L. M. Kovrizhnikh, in Plasma Physics and Controlled Nuclear Fusion (IAEA, Vienna, 1971) Vol. 1, p. 495.

10 T. H. Stix, Phys. Fluids 16,1260 (1973).

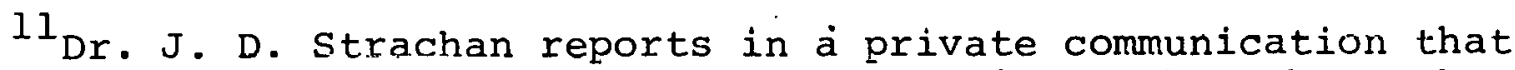
positive spikes in the loop-voltage signals have been observed during the early phase of discharges in the LT-3 Canberra tokamak.

12 H. P. Furth, J. Killeen, and M. N. Rosenbluth, Phys. Fluids $\underline{6}$, 459 (1963).

13 P. H. Rutherford, Phys. Fluids 16, 1903 (1973). 


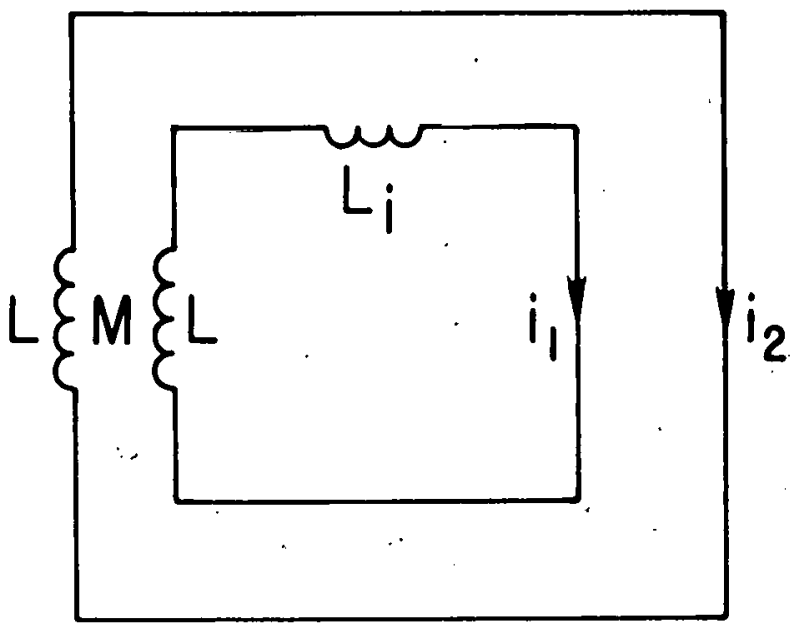

BEFORE

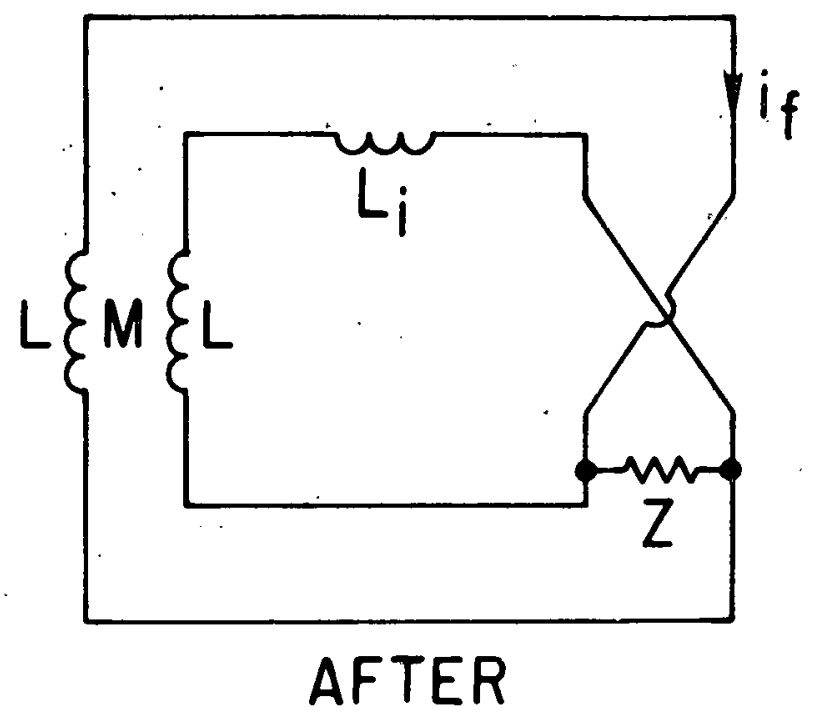

753864

Fig. 1. Circuit analogy showing reconnection of current paths following a disruption of the magnetic fields in a plasma. 

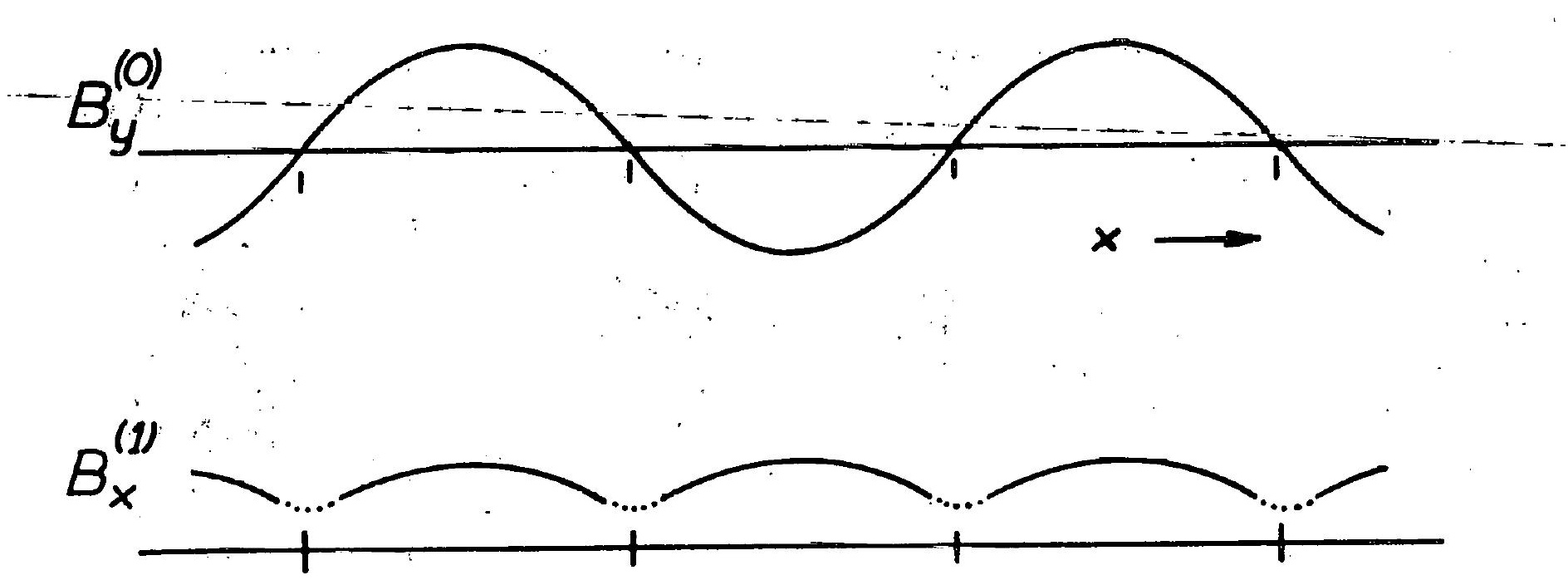

753984

Fig. 2. Linear multiple tearing mode, showing amplitudes of zero- and first-order magnetic fields. 

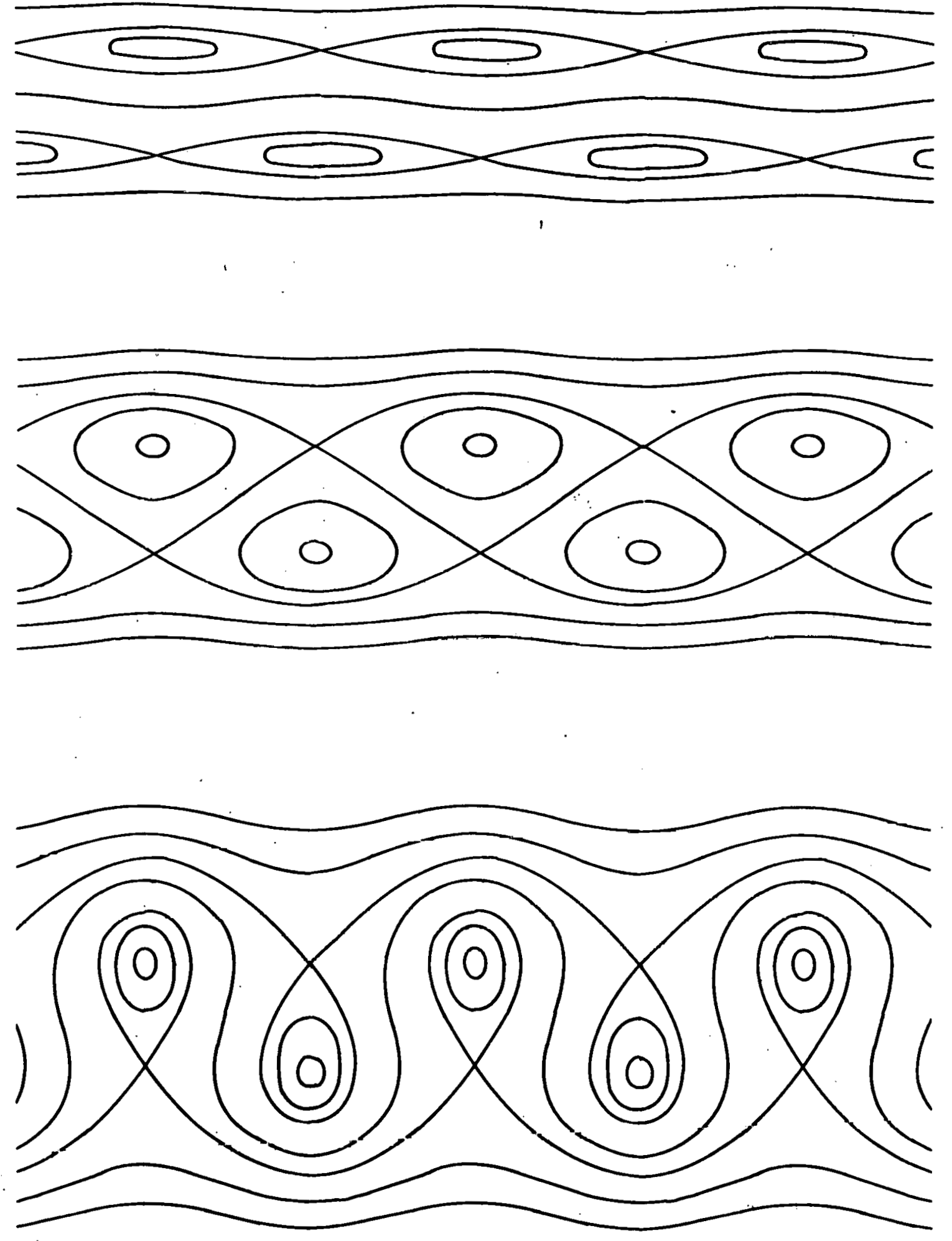

753985

-Fig. 3. Conjectured evolution of magnetic surfaces" in nonlinear double tearing instability. 